\title{
RADIOCARBON DATES OF OLD AND MIDDLE KINGDOM MONUMENTS IN EGYPT
}

\author{
Georges Bonani ${ }^{1} \bullet$ Herbert Haas $^{2} \cdot$ Zahi Hawass $^{3} \bullet$ Mark Lehner $^{4} \bullet$ Shawki Nakhla ${ }^{5}$ \\ John Nolan ${ }^{6} \bullet$ Robert Wenke $^{7} \bullet$ Willy Wölfli ${ }^{1}$
}

\begin{abstract}
Between 1984 and 1995 over 450 organic samples were collected from monuments built during the Old and Middle Kingdoms. The most suitable samples were selected for dating. The purpose was to establish a radiocarbon chronology with samples from secure context and collected with the careful techniques required for ${ }^{14} \mathrm{C}$ samples. This chronology is compared to the historical chronology established by reconstructing written documentation.
\end{abstract}

\section{INTRODUCTION}

\section{Sample Collection}

Radiocarbon dating of dynastic monuments in Egypt goes back to the very beginning of this dating method. W F Libby included three Old and Middle Kingdom samples in his initial set of known-age samples as a test of the method (Arnold and Libby 1949). In the following twenty years, numerous laboratories have followed Libby's lead and analyzed similar samples. From the published results it became apparent that close agreement with the historical chronology was often lacking. A closer study of this disagreement was needed. The American Research Center in Egypt (ARCE) undertook in 1984 the first of the two projects reported here with financial support from the Edgar Cayce Foundation. The Foundation's interest in the project rested on a hypothesis offered by Cayce that the Giza pyramids dated to $10,500 \mathrm{BC}$.

The Giza pyramids are memorials to 4th Dynasty rulers whose reigns are placed by egyptologists around $2500 \mathrm{BC}$. Our project, therefore, concentrated mostly on the Old Kingdom. The results confirmed the sequence of the monuments and their ages as they were established by historians, but the match between ${ }^{14} \mathrm{C}$ and historic dates was only approximate and left open the possibility of a difference between the two chronologies. These results were reported in Haas et al. 1987. More data was needed, thus, a second project was begun in 1995. It was designed for confirming, adjusting, or retracting the difference between the two chronologies. Support for this second project was provided by David H Koch who established the Pyramids Radiocarbon Dating Project.

In the field we looked for organic materials that were clearly linked to the construction of the monuments. Temples and pyramids built from mud bricks yielded grass, straw, and reed fragments, which were mixed into the clay and soil before shaping the bricks. Finding suitable materials in stone monuments was a greater challenge. In most of these monuments the stone building blocks were leveled and secured in place with mortar that was manufactured locally. This required massive fires to heat gypsum or limestone. The roasted minerals and the ashes from the fires were added to the mortar mix, along with remaining charcoal fragments. The usually very small fragments (1-

\footnotetext{
${ }^{1}$ Institute of Particle Physics, HPK-H30, ETH Hönggerberg, CH-8093 Zürich, Switzerland. Corresponding author. Email: bonani@particle.phys.ethz.ch.

${ }^{2}$ RC Consultants, Inc., 2846 Marida Court, Las Vegas, Nevada 89120, USA

${ }^{3}$ Undersecretary for Giza and Saqqara, Supreme Council of Antiquities, Giza Pyramids Inspectorate, Giza, Egypt

${ }^{4}$ Harvard Semitic Museum, 6 Divinity Avenue, Cambridge, Massachusetts 02186, USA

${ }^{5}$ Supreme Council of Antiquities, Abassiya, Cairo, Egypt

${ }^{6} 15$ Jay Street, Apt. 3, Cambridge, Massachusetts 02139, USA

${ }^{7}$ Department of Anthropology, University of Washington, Seattle, Washington 98195, USA
}

(C) 2001 by the Arizona Board of Regents on behalf of the University of Arizona

Near East Chronology: Archaeology and Environment. RADIOCARBON, Vol 43, Nr 3, 2001, p 1297-1320

Proceedings of the 17th International ${ }^{14} \mathrm{C}$ Conference, edited by Hendrik J Bruins, I Carmi, and E Boaretto 
$2 \mathrm{~mm}$ ) constituted the datable material. While searching the monuments, we examined seams between stone blocks for mortar filling and for black specks of charcoal inside the mortar.

Detailed records were established during both sampling projects and photographs were taken from most sampling locations. In 1984 a provenience data sheet was filled out for every sample. The samples were given a sequential three-digit number preceded by the code ARCE (American Research Center in Egypt, which provided logistic support to the project). In 1995 detailed observations on the sample and its location were entered in a field book. The samples were given three-digit numbers without a prefix. In the date list each sample can be tied to the particular project by these two distinct numbering systems, shown in column "field nr." The samples were packaged in the field and not reopened until they arrived at the dating laboratories. Loose charcoal fragments were sealed in film cans or plastic vials. Mortar pieces and mud brick fragments were wrapped in aluminum foil (or plastic wrap) and put inside a plastic bag. Labels with full provenience data were attached to each sample package.

Robert Wenke and Mark Lehner collected 76 samples in 1984. The field season began 12 December 1983 and ended 22 March 1984. Provenance details on these samples are given in Haas et al. (1987). In 1995, Robert Wenke, John Nolan, Mark Lehner, and Herbert Haas participated in the sampling effort that lasted from 26 December, 1994 until 27 February, 1995. A digest on this field season is reported in Lehner et al. (1999).

\section{Sample Pretreatment}

In spring 1984 all samples were shipped to the Southern Methodist University (SMU) ${ }^{14} \mathrm{C}$ laboratory in Dallas, Texas. During summer and fall, 64 samples were selected for dating. Pretreatment of these samples was carried out at SMU. Charcoal and fibrous samples (grass, straw, and reed) were given the usual acid-base-acid treatment. Earlier Egyptian dating projects on similar sample materials demonstrated that the integrity of charcoal was strongly degraded by all but the weakest concentrations of chemical reagents. To preserve as much sample material as possible, the treatment with base was performed with weak solutions of sodium hydroxide $(0.05$ or $0.1 \%)$. Usually, three to five such applications were made in succession until the typical brown humic acid reactions were no longer observed. Dissolving mud brick samples in distilled water and wet sieving of the slurry allowed extraction of the fibrous content. Mortar fragments were dissolved in dilute hydrochloric acid-a gradual process lasting several days. At frequent intervals the residue-sand, silt, and rare charcoal fragments-was removed and the charcoal floated off. Thirty-four samples were large enough for conventional dating (larger than $0.8 \mathrm{~g}$ of pretreated organic material) and were dated at the SMU laboratory. Thirty samples weighing $2-400 \mathrm{mg}$ were sent to the ETH laboratory for AMS dating.

There the pre-treated material was pyrolysed at about $800{ }^{\circ} \mathrm{C}$ in a pure $\mathrm{N}_{2}$ atmosphere. The pyrolysed carbon was ground, mixed with silver powder, and pressed onto a copper disc which served as target holder for the measurement (Bonani et al. 1984). Some samples were dated at both laboratories, the results of these comparison tests are given in Haas et al. (1987).

In 1995, 353 samples were collected. At the end of the collection effort these samples were divided into three groups: 1) to be dated by conventional method at the Desert Research Institute (DRI) in Las Vegas, Nevada (7 samples), 2) to be dated with AMS at the ETH laboratory in Zurich (163 samples), and 3) samples of lower priority, held in a reserve pool. The samples to be dated were sent directly to the respective laboratories. Pretreatment was handled separately at these facilities. The conventional samples received treatments similar to the details given above. 
At the ETH the samples were given the traditional acid-base-acid treatment $\left(0.5 \mathrm{M} \mathrm{HCl}\right.$ at $60^{\circ} \mathrm{C}$ for $1 \mathrm{hr}, 0.1 \mathrm{M} \mathrm{KOH}$ at $60{ }^{\circ} \mathrm{C}$ for $1 \mathrm{hr}$ and $0.5 \mathrm{M} \mathrm{HCl}$ at $60^{\circ} \mathrm{C}$ for $1 \mathrm{hr}$ ). Between the steps, the material was rinsed to $\mathrm{pH} 7$ with ultrapure, distilled water and then dried in an oven at $60{ }^{\circ} \mathrm{C}$. The samples were then combusted to $\mathrm{CO}_{2}$ for two hours at $950{ }^{\circ} \mathrm{C}$ in evacuated and sealed quartz tubes together with copper oxide and silver wire. Finally, the purified carbon dioxide was reduced in a hydrogen atmosphere to filamentous graphite over a cobalt catalyst as described by Vogel et al. (1987, 1984). The resulting graphite-cobalt mixtures were pressed onto copper discs which were used as targets in the ion source.

\section{Measurement Procedures for ${ }^{14} \mathrm{C}$}

The carbon content of conventionally dated samples was converted to benzene. ${ }^{14} \mathrm{C}$ beta decays were detected with liquid scintillation counting. Procedures for obtaining high accuracy results are described in Haas (1979); Devine and Haas (1987); Haas and Trigg (1991), Polach et al. (1987). Calculation of ${ }^{14} \mathrm{C}$ ages were performed by the standard method described in Stuiver and Polach (1977).

In 1984 , the ${ }^{14} \mathrm{C} /{ }^{12} \mathrm{C}$ and ${ }^{13} \mathrm{C} /{ }^{12} \mathrm{C}$ ratios of the samples dated with AMS were determined relative to those of secondary standards of charcoal prepared in the same way as the unknown samples. The secondary standards were normalized to the NBS oxalic acid I standard by means of high precision beta decay counting (Bonani et al. 1984). The ${ }^{14} \mathrm{C} /{ }^{12} \mathrm{C}$ and ${ }^{13} \mathrm{C} /{ }^{12} \mathrm{C}$ ratios of the 1995 batch of samples were determined relative to the NBS oxalic acid I standard values, respectively (Bonani et al. 1987). The background was determined with chemistry blank samples, which were prepared from anthracite (dead carbon) in the same way as the unknowns. All samples (unknowns, standards, and blank) of one series were measured several times (typically 3 to 4). The total measuring time per sample was confined to about 30 to 40 minutes which yielded a statistical precision of about 1-2\% in 1985 and of $0.5-0.6 \%$ per sample in 1995 . The evaluation procedure described by Stuiver and Polach (1977) was used to determine the conventional radiocarbon ages.

\section{Reporting of Sample Ages}

The report is presented in two appendices. In Appendix 1, samples from each individual monument are listed in sequence of collection, i.e. by field number and are reported as a discrete group. The dates in each group are tested for their probability of belonging to the same event, which is the construction of the monument. Chi square is used for this test. Its numerical value and the associated probability in percent are reported at the end of the sample listing for each monument, as well as the weighted mean value, the 1 sigma error and the variance. Some monuments include sample dates which are much older or younger than the established mean. Screening was used in an attempt to remove dates from samples which are probably from another context. The difference between the weighted mean of all dates and the individual dates, divided by the product of $\sqrt{2}$ and the error of the date, was used to flag outliers. Consistently eliminated were all dates where the computed number exceeded 5.0. Occasionally, several samples show as a group a distinctly different age. In such cases the samples are reported with separate mean and statistics.

The results of calibration are reported in Appendix 2. The monuments are listed in the same sequence as in the first section. The historic age range of the king who built the monument is listed, the chronology of Clayton (1994), was consulted for this information. The ${ }^{14} \mathrm{C}$ age and the error used in the calibration are stated. The error is the larger value chosen between the 1 sigma error and the variance. In this report all calibrations were performed with the calibration program developed at ETH and described in Niklaus et al. (1992). The program uses the most recent tree ring data published by Stuiver et al. (1998). For almost all monuments calibration yields several probable age 
ranges, up to five for most 4 th Dynasty monuments. Listed are all ranges resulting from a one sigma error as well as from a two sigma error. The statistical weight of each range is listed as a percent value where the sum of all range weights equals 100 percent.

Figure 1 shows the calibrated monument ages. One sigma errors were used with the averaged monument dates and every calibration range is displayed. The lengths of the solid black bars corresponds to the $\mathrm{BC}$ time span, and their width is proportional to the statistical weight of the ranges. For comparison, the historical chronology of the monuments is shown with the hatched rectangles. Applying two sigma errors to the monument dates results in wider time spans but does not significantly alter observed differences between the two chronologies.

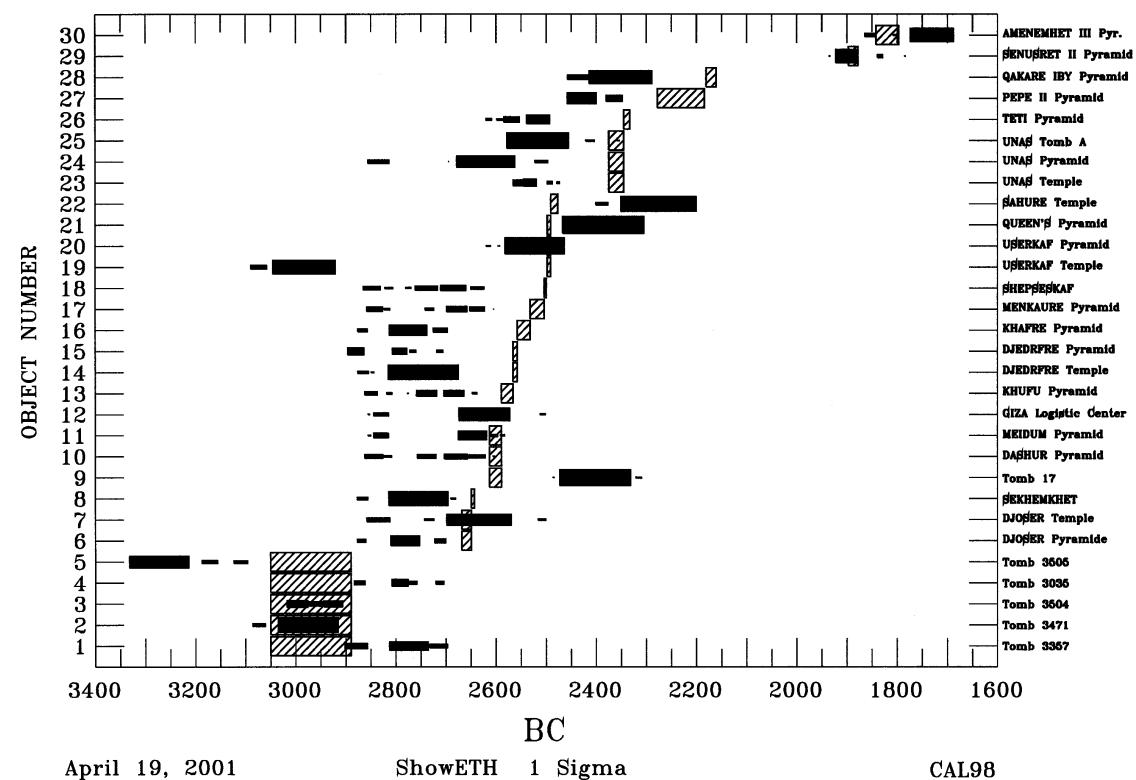

Figure 1 Comparison of the calibrated ${ }^{14} \mathrm{C}$ ranges (horizontal black bars) with the historical chronology of Clayton (1994; hatched areas). The width of the black bars is proportional to the probability of finding the true age within the corresponding one sigma range.

\section{ACKNOWLEDGMENTS}

Financial support was provided by the David H Koch Pyramids Radiocarbon Dating Project and the Edgar Cayce Foundation. Processing of the 1995 samples was assisted by Dr Irka Hajdas at ETH and by Mr Todd Enerson at DRI.

\section{REFERENCES}

Arnold JR, Libby WF. 1949. Age determinations by radiocarbon content: checks with samples of known age. Science 110(2869):678-80.

Bonani G, Balzer R, Hofmann H-J, Morenzoni E, Nessi M, Suter M, Wölfli W. 1984. Properties of milligram size samples prepared for AMS ${ }^{14} \mathrm{C}$ Dating at ETH. Nuclear Instruments and Methods in Physics Research B5:284-8.

Bonani G, Beer J, Hofmann H-J, Synal H-A, Suter M,
Wölfli W, Pfleiderer Ch, Kromer B, Junghans C, Münnich KO. 1987. Fractionation, precision, and accuracy in ${ }^{14} \mathrm{C}$ and ${ }^{13} \mathrm{C}$ measurements. Nuclear Instruments and Methods in Physics Research B29:87-90.

Clayton PA. 1994. Chronicle of the pharaohs. London: Thames and Hudson. p 224.

Devine JM, Haas H. 1987. Scintillation counter performance at the SMU radiocarbon laboratory. Radiocarbon 29(1):12-7. 
Haas H. 1979. Specific problems with liquid scintillation counting of small benzene volumes and background count rate estimation. In: Berger R, Suess H, editors. Radiocarbon dating. University of California Press. $\mathrm{p}$ 246-55.

Haas H, Trigg V. 1991. Low-level scintillation counting with a LKB Quantulus counter establishing optimal parameter settings. In: Ross H, Noakes JE, Spaulding JD, editors. Liquid Scintillation Counting and Organic Scintillators. Chelsea:Lewis Publishers. p 669-75.

Haas H, Doubrava MR. 1998. Calibration technique for ${ }^{14} \mathrm{C}$ data clusters: fitting relative chronologies onto absolute time scales. Radiocarbon 40(1):561-9.

Haas H, Devine JM, Wenke R, Lehner M, Wölfli W, Bonani G. 1987. Radiocarbon chronology and the historical calendar in Egypt. In: Aurenche O, Evin J, Hours F, editors. Chronologies in the Near East. BAR International Series 379. p 585-606.

Hassan FA, Robinson SW, 1987. High-precision radiocarbon chronometry of ancient Egypt, and comparisons with Nubia, Palestine and Mesopotamia. Antiquity 61: 119-35.

Lehner M, Nakhla S, Hawass Z, Bonani G, Wölfli W,
Haas H, Wenke R, Nolan J, Wetterstrom W. 1999. Dating the Pyramids. Archaeology 52(5):26-33.

Niklaus TR, Bonani G, Simonius M, Suter M, Wölfli W. 1992. CalibETH: an interactive computer program for the calibration of radiocarbon dates. Radiocarbon 34(3):483-92.

Polach H, Kaihola L, Haas H, Robertson S. 1987. Small sample ${ }^{14} \mathrm{C}$ dating by liquid scintillation spectrometry: Radiocarbon 30 (2):153-5.

Stuiver M, Polach H. 1977. Discussion: reporting of ${ }^{14} \mathrm{C}$ data. Radiocarbon 19(3):355-63.

Stuiver M, Reimer PJ, Braziunas TF. 1998. High-precision radiocarbon age calibration for terrestrial and marine samples. Radiocarbon 40(3):1127-51.

Vogel JS, Southon JR, Nelson DE. 1987. Catalyst and binder effects in the use of filamentous graphite for AMS. Nuclear Instruments and Methods in Physics Research B29:50-6.

Vogel JS, Southon JR, Nelson DE, Brown TA. 1984. Performance of catalytically condensed carbon for use in accelerator mass spectrometry. Nuclear Instruments and Methods in Physics Research B5:289-93. 


\section{Appendix 1: Radiocarbon Dates}

\section{$1^{\text {st }}$ Dynasty (Early Dynastic Period)}

Tomb 3357 at Saqqara

lab nr. field nr. collection site

ETH-13612 $212 \quad W$ face of $W$ wall, $~ 5 \mathrm{~m} \mathrm{~S}$ of NW corner

material

charcoal

corr. ${ }^{14} \mathrm{C}$ age

age

C14 Age

single sample

Tomb 3471 at Saqqara

BP

4222

error
1 sigma

$\delta^{13} \mathrm{C}$

permil

$-20.0$

$4222 \quad 60$

lab nr. field nr. Collection site

\section{DRl-2970 $220 \quad \sim 20 \mathrm{~m}$ S of NW corner \\ ETH-13620 $220 \quad \sim 20 \mathrm{~m}$ S of NW corner}

C14 Mean age (weighted)

all data

Chi square

material

reed

Tomb 3504 at Saqqara

lab nr. field nr. collection site

$\begin{array}{llr}\text { ETH-13626 } & 226 & \text { 9th brick course, N \& W walls } \\ \text { DRI-2968 } & 227 & \text { 9th brick course, N \& W walls } \\ \text { ETH-13627 } & 227 & \text { same sample as DRI-2968 } \\ \text { ETH-13629 } & 229 & \text { 9th brick course, N \& W walls } \\ \text { ETH-13632 } & 232 & \text { 9th brick course, N \& } W \text { walls }\end{array}$

C14 Mean age (weighted)

all data
Chi square

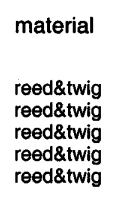

corr. ${ }^{14} \mathrm{C}$ age

$\begin{array}{rr}{ }^{4} \text { C age } & \text { error } \\ \text { y BP } & 1 \text { sigma } \\ 4236 & 57 \\ 4486 & 89 \\ 4469 & 52 \\ 4311 & 53 \\ 4319 & 56 \\ \text { mean } & 1 \text { sigma } \\ 4352 & 26 \\ & \text { probability }\end{array}$

3.1041

corr. ${ }^{14} \mathrm{C}$ a $\mathrm{Bg}$

$\begin{array}{rr}\text { y } \text { aPe } & \begin{array}{r}\text { error } \\ \text { 1 sigma }\end{array} \\ 4346 & 36\end{array}$

mean 1 sigma

$4383 \quad 30$

probability

lab nr.

ETH-13605

ETH-13610

ETH- 13608

field $\mathrm{nr}$. collection site

205 interior of W wall, mid N - S length
$210 \quad$ interior of $E$ wall, mid N - S length

210 interior of $E$ wall, mid $N$ - S length
$208 \quad W$ face of $W$ wall, between brick rows

C14 Mean age (weighted)

all data

Chi square material charcoal

charcoa

reed

BP

0.9132 corr. ${ }^{14} \mathrm{C}$ ag

y BP
4242

4242
4142

4236

mean

4210

probability

material

charcoal

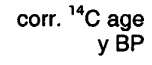

4482

BP
1 sigma

60
56

sigma

33

$\delta^{13} \mathrm{C}$

permil

-22.7
-18.2

variance

53

$19.70 \%$

$\delta^{13} \mathrm{C}$

permil

$-26.0$

$-23.2$

$-26.3$

$-16.8$

variance

46

$21.18 \%$

3505 at Saqqara

SMU-1358 ARCE 71 N - NE side of pit, from mud brick

single sample
C14 Age 


\section{$3^{\text {rd }}$ Dynasty (Old Kingdom)}

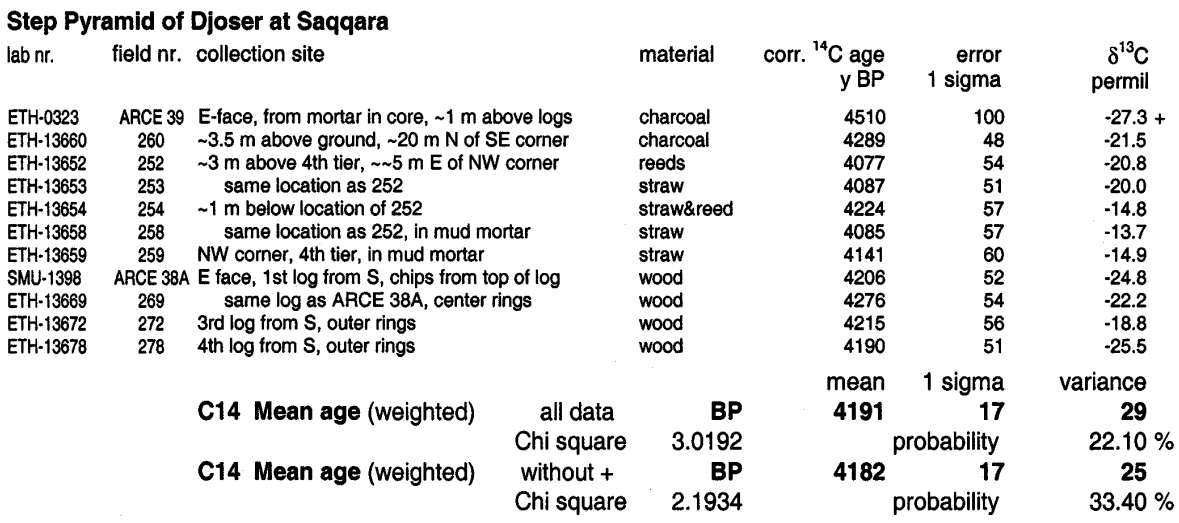

Temple Complex associated with Step Pyramid

\begin{tabular}{|c|c|}
\hline $\begin{array}{l}\text { ETH-0451 } \\
\text { ETH-0450 } \\
\text { SMU- } 1397 \\
\text { SMU-1350 } \\
\text { ETH-0231 } \\
\text { ETH-0448 } \\
\text { ETH-0449 } \\
\text { SMU-1503 } \\
\text { SMU-1362 }\end{array}$ & $\begin{array}{l}\text { ARCE } 67 B \text { eartier chapel on N side of entrance colonade } \\
\text { ARCE 67A same mud brick as ARCE } 678 \\
\text { ARCE } 40 A \text { W wall of mortuary temple, E side } \\
\text { ARCE 68B Mort. temple, E-most room, ash layer in floor } \\
\text { ARCE 68B same sample as SMU-1350 } \\
\text { ARCE 68B same sample as SMU-1350 } \\
\text { ARCE 68B same sample as SMU-1350 } \\
\text { ARCE } 69 \text { same location as ARCE } 68 B \\
\text { ARCE 70 N wall of burial shaft in S tomb, NE corner }\end{array}$ \\
\hline
\end{tabular}

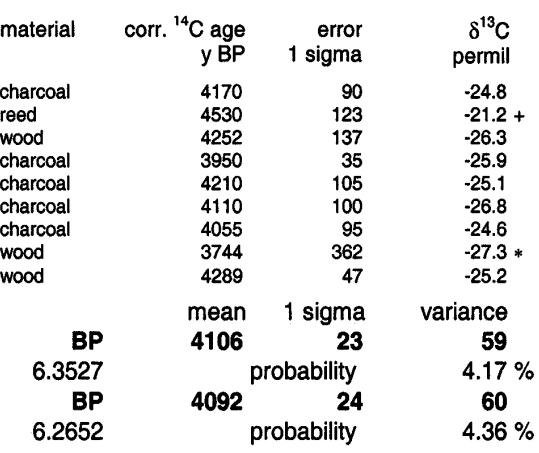

Pyramid of Sekhemkhet at Saqqara

lab nr. field nr. collection site

$\begin{array}{lcll}\text { ETH-0325 } & \text { ARCE 45A N enclosure wall, } N \text { of entrance, clay mortar } & \text { charcoal } \\ \text { ETH-13750 } & 350 \quad \text { step above entrance trench, embedded fabric } & \text { threads } \\ \text { ETH-13751 } & 351 \text { extracted with sample 350 } & \text { charcoal } \\ \text { SMU-1368 } & \text { ARCE 46B 3rd tier of masonry, from N, mud brick } & \text { grass\&straw }\end{array}$

$\begin{array}{lcr}\text { C14 Mean age (weighted) } & \text { all data } & \text { BP } \\ & \text { Chi square } & 5.9616 \\ \text { C14 Mean age (weighted) } & \text { without }+ & \text { BP } \\ & \text { Chi square } & 0.5734\end{array}$

\begin{tabular}{|c|c|c|}
\hline $\begin{array}{r}\text { corr. }{ }^{14} \mathrm{C} \text { age } \\
\text { y BP }\end{array}$ & $\begin{array}{r}\text { error } \\
1 \text { sigma }\end{array}$ & $\begin{array}{c}\delta^{13} \mathrm{C} \\
\text { permil }\end{array}$ \\
\hline $\begin{array}{l}4545 \\
4209 \\
4135 \\
4293\end{array}$ & $\begin{array}{r}80 \\
61 \\
59 \\
192\end{array}$ & $\begin{array}{l}-25.0+ \\
-26.8 \\
-26.9 \\
-23.1\end{array}$ \\
\hline $\begin{array}{l}\text { mean } \\
4254\end{array}$ & $\begin{array}{r}1 \text { sigma } \\
37 \\
\text { obability }\end{array}$ & $\begin{array}{r}\text { variance } \\
90 \\
5.08 \%\end{array}$ \\
\hline 4176 & 41 & 31 \\
\hline \multicolumn{2}{|c|}{ probability } & $75.07 \%$ \\
\hline
\end{tabular}




\section{$4^{\text {th }}$ Dynasty (Old Kingdom)}

\begin{tabular}{|c|c|c|c|c|}
\hline \multicolumn{4}{|c|}{ field nr. collection site } & material \\
\hline \multirow[t]{3}{*}{$\begin{array}{l}\text { SMU-1732 } \\
\text { ETH-13892 } \\
\text { ETH-13893 }\end{array}$} & $\begin{array}{l}\text { ARCE } 66 \mathrm{~A} \\
\quad 492 \\
493\end{array}$ & $\begin{array}{l}\text { W face, } 10 \mathrm{~m} \mathrm{~N} \text { of SW corner, } \mathrm{m} \\
\mathrm{S} \text { face, } 10 \mathrm{~m} \mathrm{E} \text { of } S W \text { comer, mu } \\
W \text { face, }-50 \mathrm{~m} \mathrm{~N} \text { of } S W \text { corner, } \mathrm{m}\end{array}$ & $\begin{array}{l}\text { brick } \\
\text { rick } \\
\text { brick }\end{array}$ & $\begin{array}{l}\text { straw } \\
\text { grass } \\
\text { grass }\end{array}$ \\
\hline & & C14 Mean age (weighted) & all data & BP \\
\hline & & C14 Mean age (weighted) & $\begin{array}{l}\text { Chi square } \\
\text { without + } \\
\text { Chi square }\end{array}$ & $\begin{array}{r}5.4209 \\
\text { BP } \\
0.0213\end{array}$ \\
\hline
\end{tabular}

\begin{tabular}{|c|c|c|}
\hline $\begin{array}{r}C \text { age } \\
\text { y BP }\end{array}$ & $\begin{array}{r}\text { error } \\
1 \text { sigma }\end{array}$ & $\begin{array}{c}\delta^{13} \mathrm{C} \\
\text { permil }\end{array}$ \\
\hline $\begin{array}{l}3978 \\
3925 \\
4195\end{array}$ & $\begin{array}{r}359 \\
55 \\
61\end{array}$ & $\begin{array}{l}-23.9 \\
-24.0 \\
-13.5+\end{array}$ \\
\hline $\begin{array}{r}\text { mean } \\
4045\end{array}$ & $\begin{array}{r}\text { sigma } \\
41\end{array}$ & $\begin{array}{r}\text { variance } \\
94\end{array}$ \\
\hline \multicolumn{2}{|c|}{ probability } & $6.65 \%$ \\
\hline 3926 & $\begin{array}{r}54 \\
\text { bability }\end{array}$ & $\begin{array}{c}8 \\
98.94 \%\end{array}$ \\
\hline
\end{tabular}

Bent Pyramid of Snefru at Dhashur

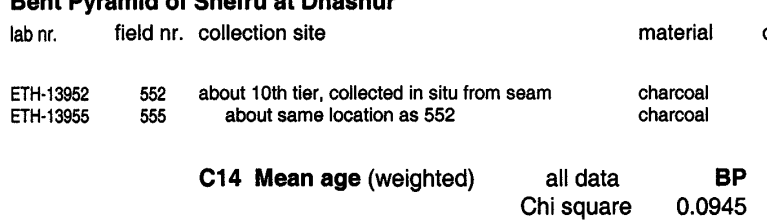

$\begin{array}{rrr}\text { corr. }{ }^{14} \mathrm{C} \text { age } & \text { error } & \delta^{13} \mathrm{C} \\ \text { y BP } & 1 \text { sigma } & \text { permil } \\ 4121 & 57 & -27.5 \\ 4146 & 58 & -25.0 \\ \text { mean } & 1 \text { sigma } & \text { variance } \\ 4133 & 41 & 12 \\ \text { probability } & 95.38 \%\end{array}$

Pyramid of Snefru at Meydum

field ni. collection site

SMU-1412
SMU-1392
ETH-13887
ETH-13888
ETH-13889
ETH-13890
ETH-13891

ar. collection site

AACE 65 burial chamber, $\log$ in E wall, outer rings

ARCE 66 E side of shaft to burial chamber, outer rings

487 burial chamber, log nr. NE comer, outer rings

488 from same log as 487

rom same log as 487

491 burial chamber, strut near ceiling, outer layer

material

corr. ${ }^{14} \mathrm{C}$ age
y BP

wood
wood
wood

wood

wood

wood

wood

C14 Mean age (weighted) all data

\section{Royal Production Center at Giza}

lab nr. field nr. collection site

$\begin{array}{llll}\text { SMU-2240 } & \text { C-3 } & \text { area A1, ash midden in East wall, above floor } & \text { charcoal } \\ \text { SMU-2275 } & \text { C-4 } & \text { area A1, feat. 15, compacted mud above floorl } & \begin{array}{l}\text { charcoal } \\ \text { charcoal }\end{array} \\ \text { ETH-5331 } & \text { C-5 } & \text { area A5, level14, site of flexed burial } & \text { charcoal } \\ \text { SMU-2274 } & \text { C-15 } & \text { area A5, East wall, below brick veneer of room } & \text { charcoal } \\ \text { ETH-5330 } & \text { C-16 } & \text { area A6, feature 16, charred disk in floor } & \text { charcoal } \\ \text { ETH-13958 } & \text { A7a-5 } & \text { from basal grey pit in unit 11 } & \text { charcoal } \\ \text { ETH-13959 } & \text { A7a-7 } & \text { from unit 33 above grey pit, acacia charcoal } & \text { charcoal }\end{array}$

C14 Mean age (weighted) all data
Chi square BP

material
charcoal
charcoal
charcoal
charcoal
charcoal
charcoal
charcoal
charcoal
BP
3.7620

\begin{tabular}{|c|c|}
\hline $\begin{array}{r}{ }^{14} \mathrm{C} \text { age } \\
\text { y BP }\end{array}$ & $\begin{array}{r}\text { error } \\
1 \text { sigma }\end{array}$ \\
\hline $\begin{array}{l}4282 \\
4232 \\
4080 \\
3982 \\
4065 \\
4205 \\
4005 \\
3985\end{array}$ & $\begin{array}{r}267 \\
49 \\
55 \\
71 \\
55 \\
45 \\
50 \\
45\end{array}$ \\
\hline $\begin{array}{r}\text { mean } \\
\mathbf{4 0 9 0}\end{array}$ & $\begin{array}{r}1 \text { sigma } \\
19 \\
\text { obability }\end{array}$ \\
\hline
\end{tabular}

$\delta^{13} \mathrm{C}$

permil

$-26.5$

-25.8
-23.7

-23.7
-27.6

$-28.1$

-25.1
-27.6

$-27.5$

variance

37 
Pyramid of Khufu at Giza

\begin{tabular}{|c|c|c|c|c|c|c|}
\hline lab nr. & field $\mathrm{nr}$. & collection site & material & corr. ${ }^{14} \mathrm{C}_{\mathrm{y} B \mathrm{BP}}$ & $\begin{array}{r}\text { error } \\
1 \text { sigma }\end{array}$ & $\begin{array}{c}\delta^{13} \mathrm{C} \\
\text { permil }\end{array}$ \\
\hline ETH-0302 & ARCE 1 & 2nd course, $\mathrm{N}$ face, $\sim 26 \mathrm{~m} \mathrm{~W}$ of NE corner & charcoal & 4260 & 80 & -24.3 \\
\hline ETH-0303 & ARCE 2 & 2nd course, $\mathrm{N}$ face, $44 \mathrm{~m} \mathrm{E}$ of $\mathrm{NW}$ comer & charcoal & 4300 & 90 & -25.4 \\
\hline ETH-0304 & ARCE 3 & 2nd course, $\mathrm{N}$ face, $\sim 60 \mathrm{~m} \mathrm{E}$ of $\mathrm{NW}$ corner & charcoal & 4245 & 85 & -24.2 \\
\hline ETH-0305 & ARCE 4 & 2nd course, $\mathrm{N}$ face, $60 \mathrm{~m} \mathrm{E}$ of $\mathrm{NW}$ comer & charcoal & 4355 & 90 & -23.5 \\
\hline ETH-4226 & ARCE 4 & same sample as ETH-0305 & charcoal & 4195 & 105 & -28.5 \\
\hline ETH-0306 & ARCE 5 & 2nd course, $\mathrm{N}$ face, $20 \mathrm{~m} \mathrm{E}$ of NW corner & charcoal & 4320 & 85 & -25.0 \\
\hline ETH-0226 & ARCE 13 & 5th course, near SE corner & charcoal & 4350 & 125 & -24.8 \\
\hline SMU-1418 & ARCE 13 & same sample as ETH-0226 & charcoal & 4258 & 273 & -26.7 \\
\hline ETH-4229 & ARCE 13 & $\begin{array}{l}\text { same sample as ETH-0226 } \\
\text { sw }\end{array}$ & charcoal & 4195 & 105 & -26.0 \\
\hline $\begin{array}{l}\text { SMU-1417 } \\
\text { ETH }-0227\end{array}$ & ARCE 14 & 5th course, S face, $\sim 5$ blocks E of SW corner & charcoal & 4359 & 241 & -25.8 \\
\hline 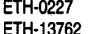 & ${ }_{362}{ }^{A}$ & same sample as SMU-1417 & charcoal & 4360 & ${ }_{58}^{125}$ & -26.6 \\
\hline $\begin{array}{l}\text { ETH-13362 } \\
\text { ETH-13754 }\end{array}$ & $\begin{array}{l}362 \\
354\end{array}$ & $\begin{array}{l}\text { 9th course, } \sim 210 \mathrm{~m} S \text { of } \mathrm{NW} \text { corner } \\
\text { 10th course, near NW corner }\end{array}$ & $\begin{array}{l}\text { charcoal } \\
\text { charcoal }\end{array}$ & 3927 & $\begin{array}{l}58 \\
57\end{array}$ & $\begin{array}{l}-24.5 \\
-22.3\end{array}$ \\
\hline ETH-13756 & 356 & $\begin{array}{l}\text { 10th course, near NW corner } \\
10 \text { th course, }-30 \mathrm{~m} \mathrm{~S} \text { of NW corner }\end{array}$ & $\begin{array}{l}\text { charcoal } \\
\text { charcoal }\end{array}$ & 4143 & 61 & -23.3 \\
\hline ETH-13757 & 357 & 10th course, $\sim 35 \mathrm{~m} \mathrm{~S}$ of NW corner & & 4225 & 79 & -26.5 \\
\hline ETH-13761 & 361 & 10th course, $200 \mathrm{~m} \mathrm{~S}$ of NW corner & charcoal & 3928 & 54 & -30.8 \\
\hline$E T H-13763$ & 363 & 10th course, above location of ETH- 13762 on 9th & charcoal & 3937 & 61 & -29.2 \\
\hline ETH-0307 & ARCE 6 & between 25 th $\& 26$ th course, $\sim 4 \mathrm{~m} \mathrm{~S}$ of NW corner & charcoal & 4440 & 90 & -21.4 \\
\hline ETH-4227 & ARCE 6 & same sample as ETH-0307 & charcoal & 4215 & 105 & -24.8 \\
\hline ETH-13770 & 370 & 49th course, $\sim 4 \mathrm{~m} \mathrm{~S}$ of NE corner & charcoal & 4087 & 53 & -26.3 \\
\hline ETH-13771 & 371 & same location as ETH-13770, \#370 & charcoal & 4187 & 60 & -25.0 \\
\hline ETH-13775 & 375 & same location as ETH-13770, \#370 & charcoal & 4190 & 52 & -28.7 \\
\hline ETH-13777 & 377 & 51 st course, $\sim 3 \mathrm{~m} \mathrm{~S}$ of NE corner & charcoal & 4313 & 57 & -23.0 \\
\hline ETH-13778 & 378 & 52nd course, near NE corner & charcoal & 4156 & 58 & -24.6 \\
\hline ETH-13779 & 379 & 52nd course, near NE corner & charcoal & 4062 & 61 & -22.7 \\
\hline ETH-0308 & ARCE 7 & 65th course, near NW corner & charcoal & 4300 & 85 & -24.1 \\
\hline ETH-4228 & ARCE 7 & same sample as ETH-0308 & charcoal & 4390 & 110 & -27.9 \\
\hline ETH-13783 & 383 & 76th course, $1.5 \mathrm{~m} \mathrm{~N}$ of SE comer & charcoal & 4237 & 62 & -19.6 \\
\hline ETH-13784 & 384 & same context as sample 383 & charcoal & 4068 & 54 & -24.8 \\
\hline ETH-13785 & 385 & same context as sample 383 & charcoal & 4083 & 53 & -27.7 \\
\hline$E T H-13782$ & 382 & 77th course, $\sim 2 \mathrm{~m} \mathrm{~N}$ of SE corner & charcoal & 3984 & 55 & -25.2 \\
\hline ETH-13787 & 387 & 81st course, 1 block $\mathrm{N}$ of SE corner & charcoal & 4197 & 49 & -26.4 \\
\hline ETH-13791 & 391 & 86th course, $1 \mathrm{~m} \mathrm{~N}$ of SE corner, surface & charcoal & 3810 & 60 & $-22.2 *$ \\
\hline ETH-0309 & ARCE 8 & 108th course, near NW comer & charcoal & 4420 & 100 & -23.9 \\
\hline ETH-13800 & 400 & 141st course, on SW corner & char & 4195 & 55 & -31.1 \\
\hline ETH-13799 & 399 & $143 \mathrm{rd}$ course, $-3 \mathrm{~m} \mathrm{E}$ of $\mathrm{SW}$ corner & charcoal & 4128 & 58 & -26.5 \\
\hline ETH-13801 & 401 & same context as sample 399 & charcoal & 4189 & 60 & -21.2 \\
\hline ETH-13802 & 402 & 145th course, $3 \mathrm{~m} \mathrm{E}$ of $\mathrm{SW}$ corner & charcoal & 4174 & 61 & -27.3 \\
\hline ETH-13803 & 403 & same context as sample 402 & char & 4062 & 60 & -25.6 \\
\hline ETH-13804 & 404 & same context as sample 402 & chat & 4254 & 59 & -27.1 \\
\hline ETH-13805 & 405 & 146th course, $1 \mathrm{~m} \mathrm{E}$ of SW comer & char & 4267 & 57 & -25.5 \\
\hline ETH-0311 & ARCE $10 \mathrm{~A}$ & 198th course, near SW corner (preserved top) & charcoal & 4395 & 85 & -24.5 \\
\hline ETH-0312 & $\begin{array}{l}\text { ARCE 10B } \\
\text { ARCE } 10 B\end{array}$ & 198th course, near SW corner (preserved top) & $\begin{array}{l}\text { charcoal } \\
\text { charcoal }\end{array}$ & 5020 & ${ }_{320}^{130}$ & $\begin{array}{l}-22.6+ \\
-19 .\end{array}$ \\
\hline $\begin{array}{l}\text { ETH-0313 } \\
\text { ETH-13900 }\end{array}$ & $\begin{array}{l}\text { ARCE } 11 \\
500\end{array}$ & $\begin{array}{l}\text { top of pyramid, on } S \text { side } \\
\text { top of pyramid, on E side }\end{array}$ & $\begin{array}{l}\text { reed } \\
\text { charcoal }\end{array}$ & $\begin{array}{l}4330 \\
4068\end{array}$ & $\begin{array}{r}125 \\
60\end{array}$ & $\begin{array}{l}-24.7 \\
-21.4\end{array}$ \\
\hline & & & & mean & 1 sigma & riance \\
\hline & & C14 Mean age (weighted) & & 4147 & 10 & 21 \\
\hline & & Chi squar & 4.2246 & & ability & $12.10 \%$ \\
\hline & & C14 Mean age (weighted) without $+\& *$ & $\begin{array}{r}\text { BP } \\
35683\end{array}$ & 4157 & $\begin{array}{r}10 \\
\text { hality }\end{array}$ & 20 \\
\hline & & & & & & $16.79 \%$ \\
\hline
\end{tabular}

Pyramid Temple of Djedefre at Abu Roash

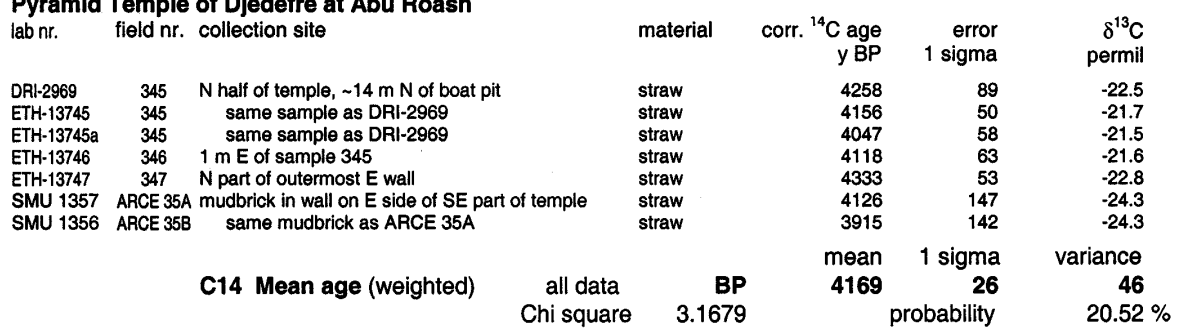


Pyramid of Djedefre at Abu Roash

lab nr. field nr. collection site

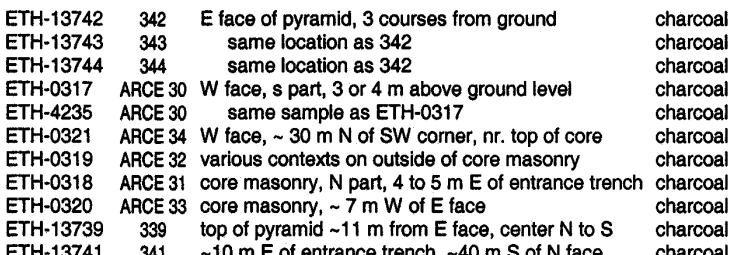

$\begin{array}{lll}\text { ETH-13739 } & 339 & \text { top of pyramid } \sim 11 \mathrm{~m} \text { from } \mathrm{E} \text { face, center } \mathrm{N} \text { to } \mathrm{S} \text { charcoal } \\ \mathrm{ETH}-13741 & 341 & \sim 10 \mathrm{~m} \mathrm{E} \text { of entrance trench, } \sim 40 \mathrm{~m} \mathrm{~S} \text { of } \mathrm{N} \text { face }\end{array}$

C14 Mean age (weighted)

all data

Chi square

BP

3.0551 corr. ${ }^{14} \mathrm{C}$ age $\quad \begin{array}{r}\text { error } \\ \text { y BP }\end{array}$

$\delta^{13} \mathrm{C}$

4073

4099

4187

4385
4330
4410
4495

4495

4230

4360
4230
4246

4246

mean

4229

probability

$\begin{array}{rr}56 & -27.7 \\ 58 & -25.2 \\ 61 & -26.5 \\ 85 & -25.0 \\ 115 & -24.1 \\ 95 & -23.7 \\ 100 & -22.6 \\ 85 & -25.0 \\ 85 & -24.6 \\ 60 & -21.5 \\ 63 & -26.1\end{array}$

1 sigma variance

38

$21.71 \%$

Pyramid of Khafre at Giza

ETH-0316 ARCE 19 1st or 2nd course, near SW corner

ETH-13822 422 betw bedrock \& 1st course, $13 \mathrm{~m} \mathrm{E}$ of SW corner

SMU-1470 ARCE 17 2nd course, 20 blocks $W$ of SE corner

ETH-4663 ARCE 17 same sample as SMU-1470

ETH 0453 ARCE 18A same general location as ARCE 17

SMU-1369 ARCE 18B $\sim 30 \mathrm{~cm}$ above location of ARCE 17

SMU-1302 ARCE 15B 3rd course $\mathrm{N}$ face, $\sim 15 \mathrm{~m}$ from passa

ETH-0314 ARCE 15C same location as ARCE 16B

ETH-13825 425 6th course, $\sim 31 \mathrm{~m} \mathrm{E}$ of SW corner

ETH-13826 426 from the same seam as sample 425

ETH-13827 427 from the same seam as sample 425

ETH-13828 428 from the same seam as sample 425

ETH-13829 429 from the same seam as sample 425

ETH-13830 430 66th course, $\sim 30 \mathrm{~m} \mathrm{E}$ of SW corner

ETH-13819 419 10th course, near SW comer

ETH-13834 434 11th course, near NE corner

ETH-13832 432 12th course, on SE corner

ETH-0315 ARCE 16 13th course, near SE corner

ETH-13837 437 13th course, near NE corner

ETH-13838 438 from the same seam as sample 437

ETH-13833 433 15th course, near SE corner

ETH-13937 537 45th course, NE comer

ETH-13936 536 46th course, $8 \mathrm{~m} \mathrm{~W}$ of NE corner

$\begin{array}{lll}\text { ETH-13936 } & 536 & 46 \text { th course, } 8 \mathrm{~m} \mathrm{~W} \text { of NE corn } \\ \text { ETH-13943 } & 543 & 84 \text { th course, near NE corner }\end{array}$

ETH-13943 543 84th course, near NE corner
ETH-0322 ARCE 37 half to $2 / 3$ way to top, SE corner

material

corr. ${ }^{14} \mathrm{C}$ age

error

1 sigma

$\delta^{13} \mathrm{C}$

charcoal

charcoal

charcoal

charcoal

charcoal

charcoal

charcoal

charcoal

charcoal

charcoal

charcoal

charcoal

charcoal

charcoal

charcoal

charcoal

charcoal

charcoal

charcoal

charcoal

charcoal

charcoal

charcoal

$\begin{array}{lcr}\text { C14 Mean age (weighted) } & \text { all data } & \text { BP } \\ & \text { Chi square } & 3.8874 \\ \text { C14 Mean age (weighted) } & \text { without }+ & \text { BP } \\ & \text { Chi square } & 3.9820\end{array}$

4500

permil

4170

4511

4511
4330
4330
4144

4165

4440

4072

4267

3975
4089

4089
4026

4129

4005

4180

4205
4235

4235
4210

4058

4058
4216

4125
4250

4250
4381

4475

mean

4174

4173

probability

probability

-23.9
-25.8

$-25.0+$

$-32.0$

$-24.8$

$-26.5$

$-27.1$

$-24.6$

$-26.4$

$-26.3$

$-28.4$

-26.9
-27.1

$-23.3$

$-26.7$

$-31.7$

$-26.7$

$-21.9$

$-26.9$

$-27.3$

$-24.4$

-27.9
-18.6

$-18.6$

$-23.9$

variance

26

$14.32 \%$

27

$13.66 \%$

Sphinx Temple of Khafre at Giza 1)

lab nr. field nr. collection site

material
charcoal
charcoal

corr. ${ }^{14} \mathrm{C}$ age

$\delta^{13} \mathrm{C}$

permil

-26.9
-26.9

ETH-0228 ARCE $20 \mathrm{~S}$ side of eastern columned recess

SMU-1416 ARCE 20 same sample as ETH-0228

charcoal

y BP

1 sigma

$-25.8$ 


\section{Pyramid of Menkaure at Giza}

labnr. field nr. collection site

\begin{tabular}{|c|c|c|}
\hline $\begin{array}{l}\text { ETH-13850 } \\
\text { ETH-4232 }\end{array}$ & $\begin{array}{l}450 \\
\text { ARCE } 21\end{array}$ & $\begin{array}{l}\text { 6th course, } \sim 15 \mathrm{~m} \mathrm{~S} \text { of NE corner } \\
\sim 9 \text { th course ( } \sim \text { th above granite casing) } E \text { face }\end{array}$ \\
\hline ETH-13852 & 452 & $\begin{array}{l}\text { 10th course, } 4 \mathrm{~m} \mathrm{~S} \text { of NE corner } \\
\text {. }\end{array}$ \\
\hline ETH-13853 & 453 & 12th course, $15 \mathrm{~m} \mathrm{~S}$ of $\mathrm{NE}$ corn \\
\hline ETH-13854 & 454 & 12th course, $\sim 15 \mathrm{~m} \mathrm{~S}$ of NE \\
\hline ETH-13855 & 455 & same location as 454 \\
\hline ETH-13857 & & 13th course, $\sim 18 \mathrm{~m} \mathrm{~S}$ of NE corner \\
\hline ETH-0454 & ARCE22/1 & $\begin{array}{l}15 \text { th course, near SE corner } \\
\text { 1 }\end{array}$ \\
\hline ETH-0455 & ARCE22/2 & same sample as ARCE 22/1 \\
\hline $\mathrm{TH}-4233$ & ARCE 22B & same location as ARCE 22/1 \\
\hline ETH-13859 & 459 & 16th course, $\sim 5 \mathrm{~m} \mathrm{~S}$ of NE corner \\
\hline-1386 & 461 & 18th course,, $5 \mathrm{~m} \mathrm{~S}$ of $\mathrm{NE}$ corner \\
\hline H-1386 & 462 & in same mortar seam as 461 \\
\hline ETH-13863 & 463 & in same mortar seam as 461 \\
\hline ETH-13864 & 464 & in same mortar seam as 461 \\
\hline ETH-13865 & 465 & in same mortar seam as 461 \\
\hline ETH-13867 & 467 & 18th course, $\sim 3.5 \mathrm{~m} \mathrm{~S}$ of NE corner \\
\hline ETH-13868 & 468 & 21st course, $\sim 10 \mathrm{~m} \mathrm{~W}$ of NE corner \\
\hline ETH-13869 & 469 & 24th course, near NE corner \\
\hline ETH-13871 & 471 & 27th course, near NE corner \\
\hline SMU-1370 & ARCE 23 & 37th course, $E$ face near $S E$ corner \\
\hline ETH-4234 & ARCE 23 & same sample as SMU-1370 \\
\hline ETH-1391C & 510 & 45th course, near NE comer \\
\hline ETH-13911 & 511 & 48th course, near NE comer \\
\hline SMU-1415 & ARCE 25 & 54th course, $S$ face, 2 blocks $W$ of $S E$ corner \\
\hline ETH-0229 & ARCE 25 & same sample as SMU-1415 \\
\hline $\begin{array}{l}\text { ETH-13914 } \\
\text { ETH-13915 }\end{array}$ & 514 & $\begin{array}{l}\text { 56th course, E face near SE corner } \\
57 \text { th course E face near SE comer }\end{array}$ \\
\hline ETH-13918 & 518 & $\begin{array}{l}57 \text { th course, E face near SE corner } \\
70 \text { th course, E face near SE corner }\end{array}$ \\
\hline ETH-13919 & 519 & 70th course, $\mathrm{E}$ face near SE corner \\
\hline SMU-1352 & ARCE & 58th course, near SE corner, gap betw. blocks \\
\hline SMU-1419 & ARCE & 3 same as ARCE $26 \mathrm{~A}$ \\
\hline SMU-1414 & ARCE 27A & A same general location, below b \\
\hline & & Ec \\
\hline ETH-13919 & & ot \\
\hline
\end{tabular}

\begin{tabular}{|c|c|c|c|}
\hline material & $\begin{array}{r}\text { corr. }{ }^{14} \mathrm{C} \text { age } \\
\text { y BP }\end{array}$ & $\begin{array}{r}\text { error } \\
1 \text { sigma }\end{array}$ & $\begin{array}{c}\delta^{13} \mathrm{C} \\
\text { permil }\end{array}$ \\
\hline charcoal & 4335 & 60 & -27.0 \\
\hline charcoal & 4145 & 105 & -25.0 \\
\hline charcoal & 4489 & 54 & $-23.5+$ \\
\hline char & 4074 & 47 & -25.2 \\
\hline charcoal & 4236 & 53 & -22.0 \\
\hline char & 3917 & 48 & -28.0 \\
\hline ai & 39 & 64 & -26.5 \\
\hline charcoal & 44 & 105 & -22.8 \\
\hline arcoal & 4310 & 105 & $\begin{array}{l}-28.0 \\
-289\end{array}$ \\
\hline larcoal & 4245 & 95 & $\begin{array}{l}-28.9 \\
-27.6 *\end{array}$ \\
\hline tarcoal & 3803 & 63 & $\begin{array}{l}-27.6 * \\
-26.7\end{array}$ \\
\hline charcoal & 3833 & $\begin{array}{l}62 \\
55\end{array}$ & $\begin{array}{l}-26.7 \\
-27.1\end{array}$ \\
\hline charcoal & $\begin{array}{l}3939 \\
3994\end{array}$ & $\begin{array}{l}55 \\
54\end{array}$ & $\begin{array}{l}-27.1 \\
-26.5\end{array}$ \\
\hline $\begin{array}{l}\text { charcoal } \\
\text { charcoal }\end{array}$ & 4226 & 54 & -26.4 \\
\hline $\begin{array}{l}\text { Charcoal } \\
\text { charcal }\end{array}$ & 4060 & 54 & -26.6 \\
\hline char & 4082 & 55 & $\begin{array}{l}-26.0 \\
\end{array}$ \\
\hline char & 4319 & 61 & -25.1 \\
\hline charcoal & 4115 & 53 & -25.1 \\
\hline charcoal & 4163 & 56 & -23.4 \\
\hline charcoal & 4048 & 48 & -25.9 \\
\hline charcoal & 4180 & 90 & -29.1 \\
\hline charcoal & 4062 & 49 & -26.7 \\
\hline charcoal & 4186 & 59 & -25.5 \\
\hline charcoal & 4418 & 250 & -26.1 \\
\hline charcoal & 4310 & 135 & -22.0 \\
\hline charcoal & 4445 & 67 & -23.4 \\
\hline charcoal & 4188 & 59 & -24.4 \\
\hline charcoal & 4257 & 60 & -24.5 \\
\hline charcoal & 4122 & 51 & -26.6 \\
\hline grown powder & 3735 & 59 & $-21.9 *$ \\
\hline brown powder & 3685 & 60 & $-21.9 *$ \\
\hline brown powder & 3768 & 51 & $-20.9 *$ \\
\hline charcoal & 4257 & 60 & -24.5 \\
\hline charcoal & 4122 & 51 & -26.6 \\
\hline & mean & 1 sigma & variance \\
\hline BP & 4132 & 11 & 29 \\
\hline 7.2814 & \multicolumn{2}{|c|}{ probability } & $2.62 \%$ \\
\hline BP & 4127 & 11 & 25 \\
\hline 5.3294 & & robability & $6.96 \%$ \\
\hline
\end{tabular}

Mortuary Temple of Shepseskaf at South Saqqara lab nr. field $\mathrm{nr}$. collection site

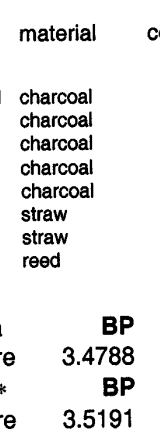

corr.

\begin{tabular}{rr}
${ }^{14} \mathrm{C}$ age & error \\
y BP & 1 sigma \\
4041 & 60 \\
4014 & 58 \\
3769 & 208 \\
4380 & 150 \\
4265 & 138 \\
4220 & 59 \\
4319 & 63 \\
4101 & 59 \\
mean & 1 sigma \\
4140 & 26 \\
\multicolumn{3}{c}{ probability } \\
4146 & 26 \\
\multicolumn{2}{c}{ probability }
\end{tabular}

$\delta^{13} \mathrm{C}$
permil
-22.6
-26.1
$-27.0 *$
-25.9
-25.7
-22.4
-5.3
-22.0
ariance
48
$17.56 \%$
48
$17.21 \%$


Mastaba el-Faraoun of Shepseskaf at South Saqqara 2) lab nr. field nr. collection site

SMU-1371 ARCE 54B 1st course on platform, 18 blocks W of SE corner

ETH-0230 ARCE 54C same location as ARCE 54B

SMU-1396 ARCE 54C same location as ARCE 54B

ETH-13731 331 3rd course, $20 \mathrm{~m}$ W of SE corner

ETH-0329 ARCE 56 1st course, core masonry from $E$ and $W$ faces

\begin{tabular}{|c|c|c|c|}
\hline material & $\begin{array}{r}\text { corr. }{ }^{14} \mathrm{C} \text { age } \\
\text { y BP }\end{array}$ & $\begin{array}{r}\text { error } \\
1 \text { sigma }\end{array}$ & $\underset{\text { permil }}{\delta^{13} \mathrm{C}}$ \\
\hline $\begin{array}{l}\text { charcoal } \\
\text { charcoal } \\
\text { charcoal } \\
\text { wood } \\
\text { charcoal }\end{array}$ & $\begin{array}{l}3330 \\
4350 \\
3792 \\
4086 \\
4320\end{array}$ & $\begin{array}{r}56 \\
170 \\
34 \\
57 \\
80\end{array}$ & $\begin{array}{l}-26.8 \\
-20.1 \\
-25.8 \\
-23.8 \\
-23.5\end{array}$ \\
\hline BP & $\begin{array}{r}\text { mean } \\
\mathbf{3 8 1 9}\end{array}$ & $\begin{array}{r}1 \text { sigma } \\
24\end{array}$ & $\begin{array}{r}\text { variance } \\
148\end{array}$ \\
\hline
\end{tabular}

\section{$5^{\text {th }}$ Dynasty (Old Kingdom)}

\section{South Pyramid Temple of Userkaf at Saqqara}

lab nr. field nr. collection

ETH-0326 ARCE 51 bedding of basalt paving blocks, morta

SMU-1495 ARCE 51 same sample as ETH-032

ETH-4236 ARCE 51 same sample as ETH-0326

ETH-0327 ARCE 52 bedding of basalt paving blocks, mortar

ETH-4237 ARCE 52 same sample as ETH-0326

ETH.0328 ARCE 53 bedding of basalt paving blocks, mortar

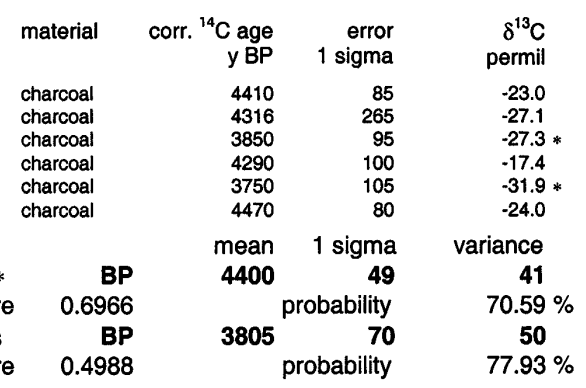

\section{C14 Mean age (weighted) \\ C14 Mean age (weighted)

0.4988

\section{Pyramid of Userkaf at Saqqara}

lab nr. field nr. collection site

ETH-13706

ETH -13707
ETH- 13703 s

ETH-13704

ETH- 13710

ETH-13714

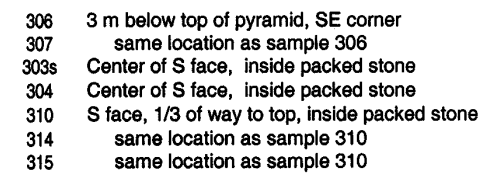

material

charcosil

charcoal

reed 3)

reed 4)

reed 5)

charcoa

charcoal

C14 Mean age (weighted) without *

Chi square

C14 Mean age (weighted), $3 *$ dates corr. ${ }^{14} \mathrm{C}$ age

y BP

4067

3990

2521

2589

2432

4114
3859

mean

4009

2512

9068

BP

2.3001 error

1 sigma

57

57
56

53

51
55

1 sigma

probability

probability $\underset{\delta^{13} \mathrm{C}}{\text { permil }}$

$-26.7$

$-24.8$

$-22.5 *$

$-18.9 *$

$-26.2 *$

-28.7
-25.2

variance

56

$14.18 \%$

46

$31.66 \%$

Queen's Pyramid of Userkaf at Saqqara

lab nr. field nr. collection site

SMU-1413 ARCE 50 E of entrance passage, below core blocks

ETH-13703 303 mudbrick from wall, SE of Quenn's Pyramid

ETH-13703a 303a same mudbrick as 303

corr. ${ }^{14} \mathrm{C}$ age

error

1 sigma

reed\&wood 6)

charcoal 7)

charcoal

C14 Mean age (weighted)

without *

Chi square

BP

0.4858 y BP
3985

2498

2498

mean

3905 probability

$\delta^{13} \mathrm{C}$

permil

$-23.7$

$-23.4$

variance

41

$78.43 \%$ 


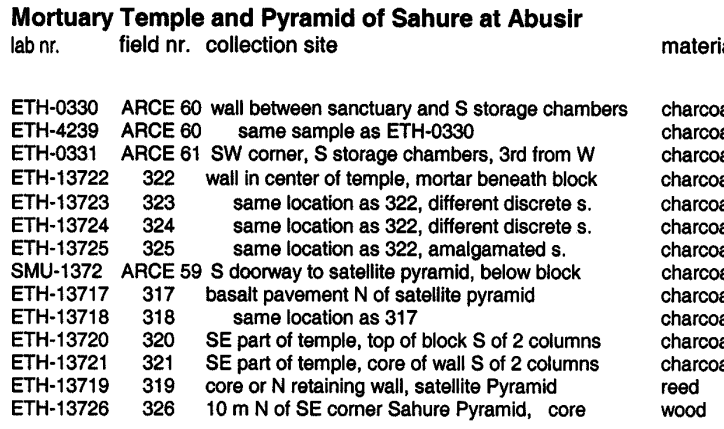

$\begin{array}{lcr}\text { C14 Mean age (weighted) } & \text { all data } & \text { BP } \\ & \text { Chi square } & 22.1344 \\ \text { C14 Mean age (weighted) } & \text { without }+\& * & \text { BP }\end{array}$

Chi square $\quad 7.7737$

\begin{tabular}{|c|c|c|}
\hline $\begin{array}{r}\text { corr. }{ }^{14} \mathrm{C} \text { age } \\
\text { y BP }\end{array}$ & $\begin{array}{r}\text { error } \\
1 \text { sigma }\end{array}$ & $\begin{array}{r}\delta^{13} \mathrm{C} \\
\text { permil }\end{array}$ \\
\hline $\begin{array}{l}4260 \\
3925 \\
3400 \\
3447 \\
3647 \\
3618 \\
3760 \\
4042 \\
3918 \\
4042 \\
4003 \\
3942 \\
3821 \\
4397\end{array}$ & $\begin{array}{r}85 \\
125 \\
85 \\
56 \\
50 \\
50 \\
51 \\
99 \\
59 \\
51 \\
59 \\
58 \\
59 \\
51\end{array}$ & $\begin{array}{l}-27.7+ \\
-28.8 \\
-20.0 * \\
-26.7 * \\
-26.3 \\
-26.0 \\
-25.1 \\
-26.8 \\
-26.6 \\
-23.7 \\
-22.9 \\
-23.1 \\
-9.6 \\
-17.8+\end{array}$ \\
\hline $\begin{array}{r}\text { mean } \\
3862\end{array}$ & $\begin{array}{r}1 \text { sigma } \\
16 \\
\text { obability }\end{array}$ & $\begin{array}{r}\text { variance } \\
76 \\
0.00 \%\end{array}$ \\
\hline 3840 & $\begin{array}{r}19 \\
\text { bability }\end{array}$ & $\begin{array}{c}52 \\
2.05 \%\end{array}$ \\
\hline
\end{tabular}

material

corr. ${ }^{14} \mathrm{C}$ age

y BP
4058

4058
4036

4040

3921

4004

4041

mean

4009

probability

material
charcoal
charcoal
charcoal
straw
charcoal

BP
2.4547

corr. ${ }^{14} \mathrm{C}$ age

error

sigma

4255

4101
4290

4290
4035

4035
4015

mean

4079

probability

material

corr. ${ }^{14} \mathrm{C}$ age

1 sigma

sigma

3924

mean

3981

probability $\delta^{13} \mathrm{C}$
permil
-25.5
-27.0
-25.1
-25.1
-25.9
-25.9
variance
22
$61.57 \%$

$\delta^{13} \mathrm{C}$

permil

-25.9
-27.7

$-22.9$

$-3.8$

variance

50

$29.31 \%$

$\delta^{13} \mathrm{C}$

permil

-24.6
-22.1

variance

55 


\section{$6^{\text {th }}$ Dynasty (Old Kingdom)}

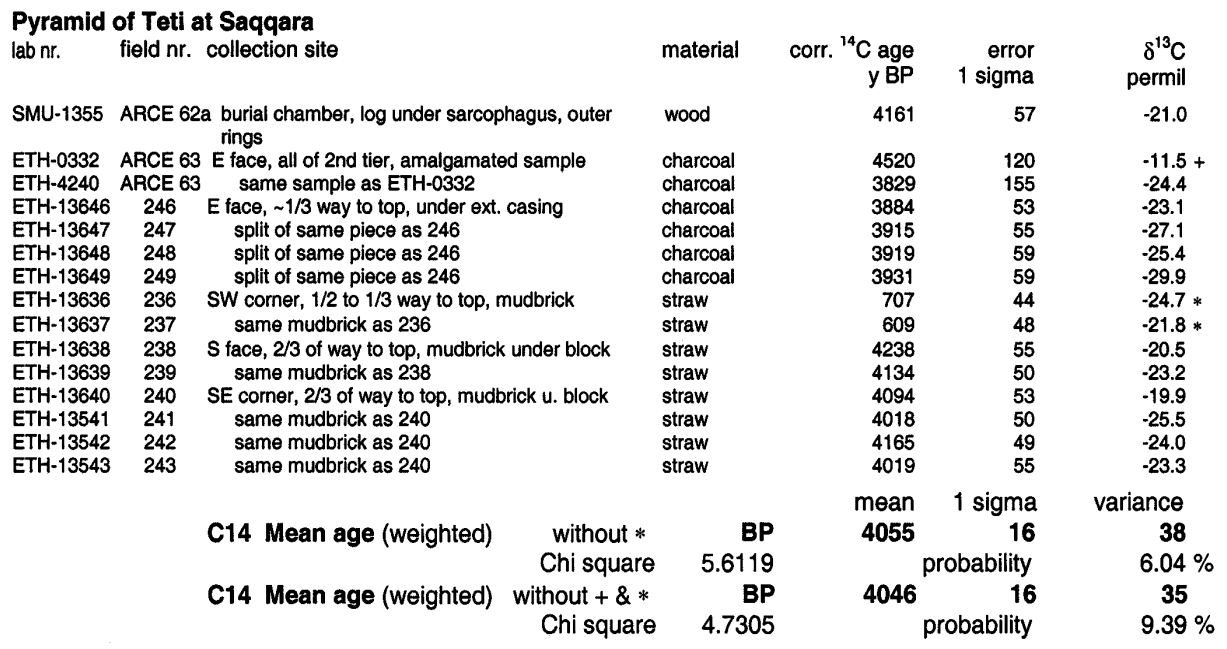

Mortuary Chapel of Queen Neith (reign of Pepi II) at Saqqara 8)

lab nr. field $\mathrm{nr}$. collection site material ETH-4238 ARCE bedding of pavement

SMU-1469 ARCE 57a same context as ARCE 57, larger pieces

Pyramid of Pepi II at Saqqara

lab nr. field nr. collection site

SMU-1351 ARCE 58 S face, 1st course, amalgamated sample

C14 Age

single sample charcoal

charcoal

material

charcoal

BP corr. ${ }^{14} \mathrm{C}$ ag

y BP

corr. ${ }^{14} \mathrm{C}$ age

error

1 sigma

135

140

error

1 sigma

24

3900 $\delta^{13} \mathrm{C}$

$-23.8$

$-26.6$

$\delta^{13} \mathrm{C}$

permil

$-26.4$

\section{$8^{\text {th }}$ Dynasty (First Intermediate Period)}

\section{Pyramid of Qakare-lby at South Saqqara}

lab nr. field nr. collection site

ETH-13728 328 core of pyramid, mudbrick

C14 Age

single sample

material
straw

$\quad$ BP

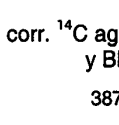

erro

1 sigma

$\delta^{13} \mathrm{C}$

permil

$-17.2$ 


\section{$12^{\text {th }}$ Dynasty (Middle Kingdom)}

Pyramid of Amenemhet I at Lisht 9)

ETH-13885

material
straw
charcoal
BP
0.1448

corr. ${ }^{14}$

$\begin{array}{rrr}{ }^{14} \mathrm{C} \text { age } & \text { error } & \delta^{13} \mathrm{C} \\ \text { y BP } & 1 \text { sigma } & \text { permil } \\ 2916 & 50 & -24.1 \\ 2944 & 54 & -23.9 \\ \text { mean } & 1 \text { sigma } & \text { variance } \\ 2929 & 37 & 14 \\ & \text { probability } & 93.02 \%\end{array}$

Pyramid of Senusret II at Illahun

lab nr. field nr. collection site

DRI-2947

ETH-13924

ETH-13925

ETH-13926

ETH-13927

ETH-13928

ETH-13931

ETH-13932

DRI-2971

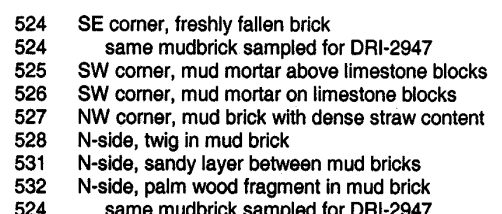

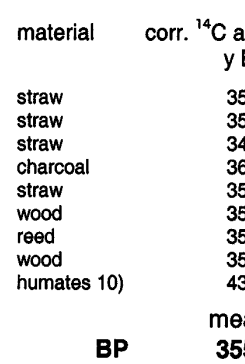

C14 Mean age (weighted)

$$
\begin{aligned}
& \text { without }+ \\
& \text { Chi square }
\end{aligned}
$$

0.6893

$\begin{array}{rr}\begin{array}{rr}\text { C age } \\ \text { y BP }\end{array} & \begin{array}{r}\text { error } \\ \text { s sigma }\end{array} \\ 3580 & 104 \\ 3545 & 51 \\ 3488 & 54 \\ 3641 & 55 \\ 3538 & 54 \\ 3527 & 54 \\ 3582 & 52 \\ 3534 & 58 \\ 4342 & 70 \\ \text { mean } & \text { 1 sigma } \\ 3552 & 20 \\ & \text { probability }\end{array}$

$\delta^{13} \mathrm{C}$
permil
-25.1
-24.6
-24.0
-28.7
-28.0
-11.1
-9.5
-19.8
$-19.7+$
variance
17
$70.85 \%$

Pyramid of Amenemhet III at Dashur

lab nr.

field $\mathrm{nr}$. collection site

material corr. ${ }^{14} \mathrm{C}$ age

$\begin{array}{rr}\begin{array}{r}\text { error } \\ 1 \text { sigma }\end{array} & \begin{array}{r}\delta^{13} \mathrm{C} \\ \text { permil }\end{array} \\ 41 & -24.2 \\ 73 & -19.6\end{array}$

DRI-2948
DRI-2958

556

mudbrick from the pyramid

same mudbrick sampled for DRI-2948

straw

humates 10) $\quad 4452$

41

\section{Remarks and Footnotes}

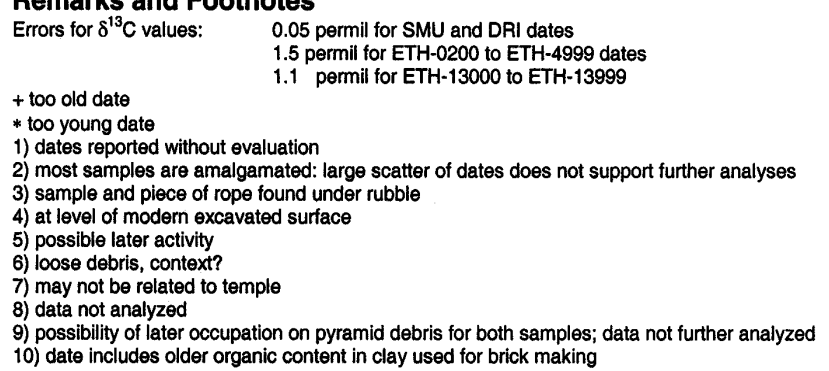




\section{Appendix 2: Calibrated Dates}

\section{$1^{\text {st }}$ Dynasty (Early Dynastic Period)}

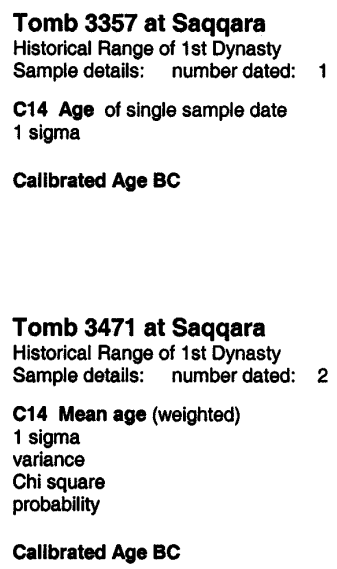

Tomb 3035 at Saqqara Historical Range of 1st Dynasty Sample details: number dated: 3

C14 Mean age (weighted)

1 sigma

Chi square

Callibrated Age BC

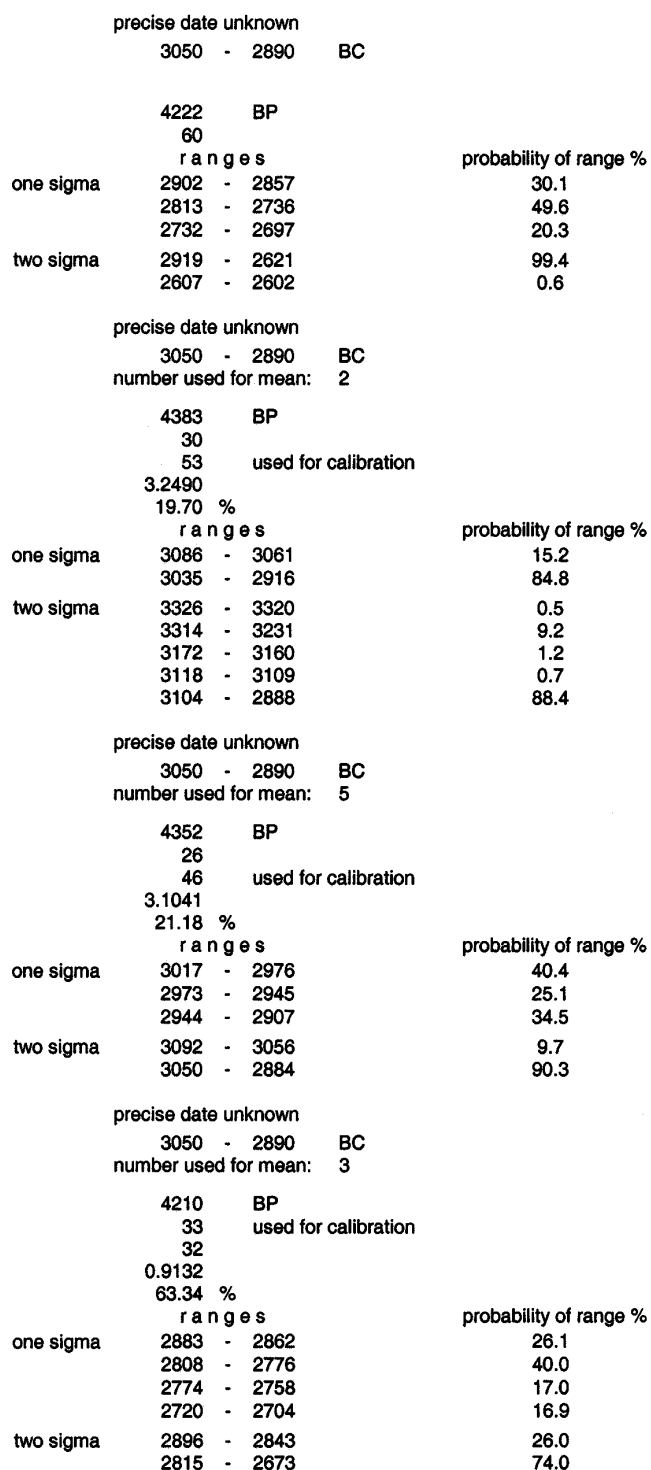

one sigma

two sigma 
Tomb 3505 at Saqqara

Historical Range of 1st Dynasty Sample details: number dated:

C14 Age of single sample date

1 sigma

Calibrated Age BC

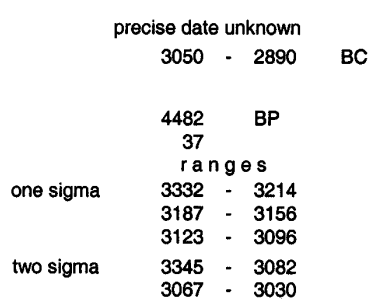

probability of range $\%$

68.0

16.9

15.0

91.8

\section{$3^{\text {rd }}$ Dynasty (Old Kingdom)}

\section{Step Pyramid of Djoser at Saqqara} Historical Rang

Sample details: number dated: 11

$2668-2649 \quad B C$

C14 Mean age (weighted)

1 sigma

variance

Chi square
probability

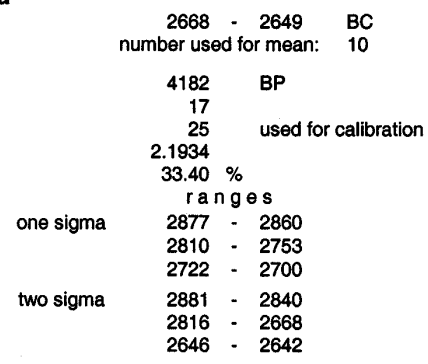

Calibrated Age BC

probability of range \%

18.3
58.5

23.2

18.9

80.3
0.8

Temple Complex associated with Step Pyramid

Historical Range

Sample details: number dated: 9

C14 Mean age (weighted)

1 sigma

Chi square

probability

Calibrated Age BC

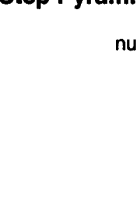

2668 - $2649 \quad B C$ number used for mean: $\quad 7$

4092 BP

60 used for calibration

6.2652

$4.36 \%$

\begin{tabular}{ll} 
& \multicolumn{2}{c}{ r a nges } \\
one sigma & $2858 \div 2812$ \\
& $2743 \div 2724$
\end{tabular}

2698 - 2570

$2516 \div 2501$

two sigma 2872 - 2800

2784 - 2547

2544 - 2489

2479 - 2474

probability of range $\%$

22.1
7.7

63.5

20.2

67.2

11.9

$2649-2643 \quad B C$

Pyramid of Sekhemkhet at Saqqara

Historical Range

Sample details: number dated: 4

C14 Mean age (weighted)

1 sigma

Chi square

probability

Calibrated Age BC

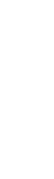

one sigma

\begin{tabular}{|c|c|}
\hline 4176 & BP \\
\hline 41 & used for calibration \\
\hline 31 & \\
\hline 0.5734 & \\
\hline $\begin{array}{r}75.07 \\
\mathrm{ra}\end{array}$ & \\
\hline 2877 & - 2856 \\
\hline 2814 & - 2696 \\
\hline 2690 & - 2681 \\
\hline $\begin{array}{l}2882 \\
2823\end{array}$ & $\begin{array}{r}-\quad 2828 \\
-\quad 2658\end{array}$ \\
\hline 2652 & 2623 \\
\hline
\end{tabular}

probability of range $\%$

14.9

79.7

5.3

20.2
72.2

7.3 


\section{$4^{\text {th }}$ Dynasty (Old Kingdom)}

\section{Tomb 17, reign of Snefru at Meydum}

Historical Rang

Historical Range

C14 Mean age (weighted)

1 sigma

variance

Chi square

probability

Calibrated Age BC

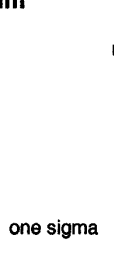

one sigma

two sigma

Bent Pyramid of Snefru at Dashur Historical Range Sample details: number dated: 2

C14 Mean age (weighted)

1 sigma

Chi square
probability

Calibrated Age BC $\begin{array}{cl}2613 & -2589 \\ \text { number used for mean: } & \text { BC }\end{array}$

umber used for me

3926 BP

54 used for calibration

0.0213

$98.94 \%$

ranges

$2486 \cdot 2485$

2473 - 2332

2321 - 2310

2570 - 2516

2501 - 2280

2219 - 2209

2613 - 2589 BC

number used for mean: 2

4133 BP

41 used for calibration

0.0945

$95.38 \%$

ranges

one sigma 2862 - 2826

2824 - 2809

2757 - 2720

2703 - 2657

$2703-2657$
$2653-2622$

2606 - 2603

2875 - 2797

2788 - 2617

two sigma

2612 - 2581

Pyramid of Snefru at Meydum

Historical Range

Sample details

C14 Mean age (weighted)

1 sigma

variance

Chi square

probability

Calibrated Age BC

2613 - $2589 \quad B C$ number used for mean: 6

$\begin{array}{cccc} & 4110 & \text { BP } \\ & 23 & \text { used for calibration } & \\ 17 & & \\ & 0.5618 & \\ & 75.51 \% & \\ \text { one sigma } & \text { r a } \mathrm{g} \text { g } \mathrm{s} & \\ & 2855-2850 & 27.8 \\ & 2844-2815 & 50.4 \\ & 2675-2619 & 11.7 \\ & 2610-2597 & 6.3 \\ \text { two sigma } & 2591-2583 & 27.8 \\ & 2860-2810 & 8.4 \\ & 2753-2722 & 46.6 \\ & 2700-2616 & 17.1\end{array}$

Royal Production Center at Giza Historical Range not established

Sample details: number dated: 8

C14 Mean age (weighted)

1 sigma

Chi square

probability number used for mean: 8

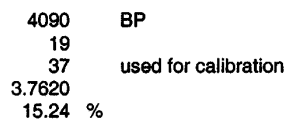

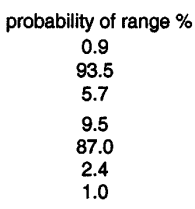

probability of range $\%$

21.5

9.1

21.2

27.6
18.6

1.9

63.5

8.2

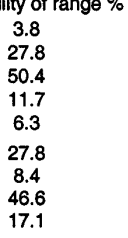




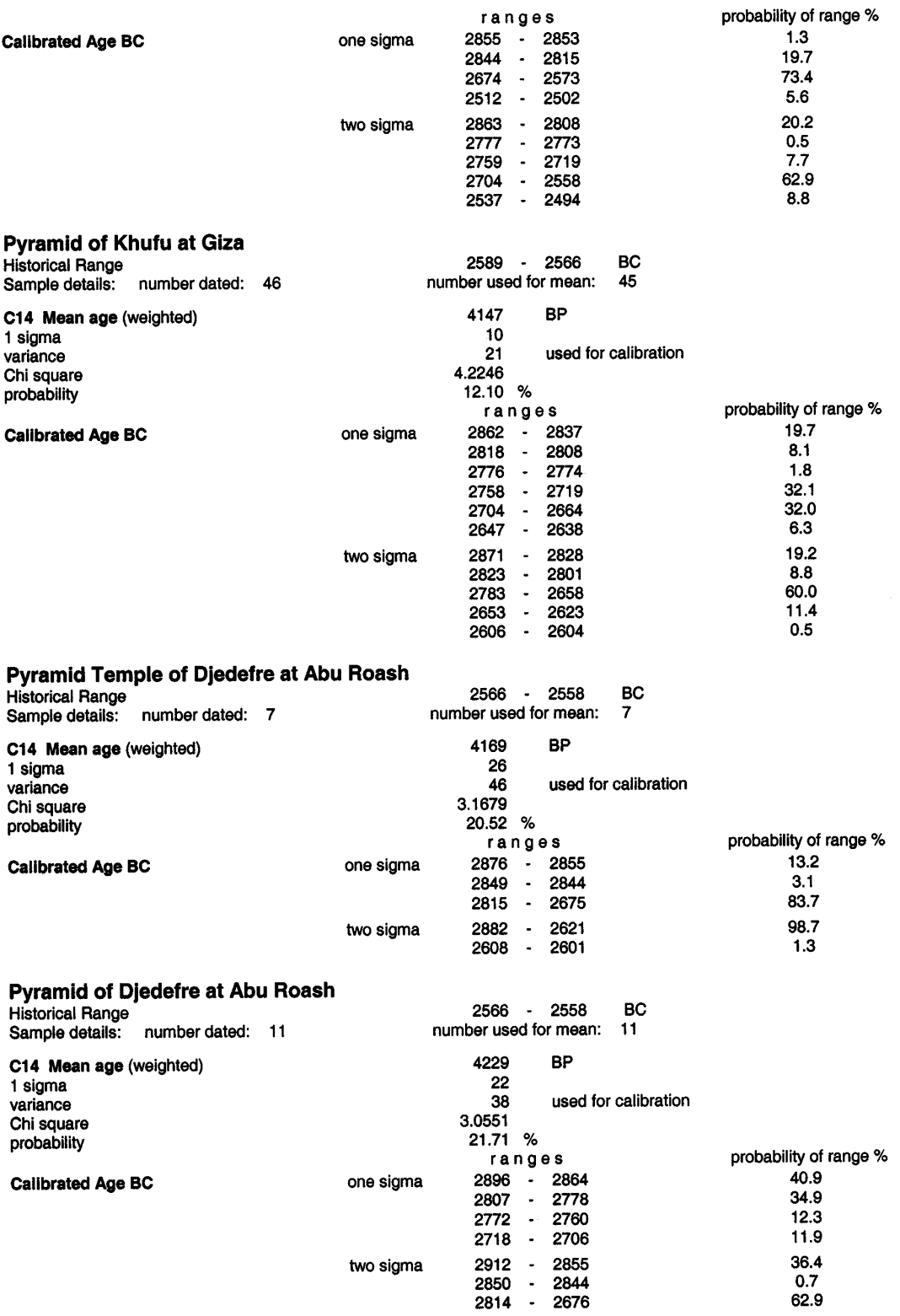




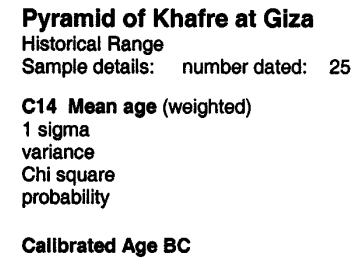

yramid of Menkaure at Giza Historical Range Sample details: number dated: 35

C14 Mean age (weighted)

1 sigma

Chi square

probability

Calibrated Age BC

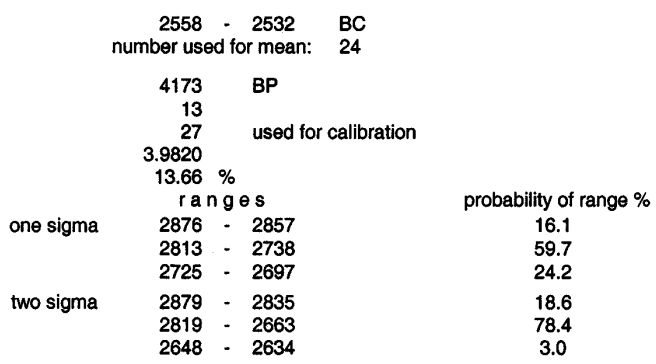

$2532-2504$ BC number used for mean: $\quad 30$

4127 BP

11

25 used for calibration

$5.3294 \%$
$6.96 \%$

ranges

2858 - 2827

2823 - 2812

2741 - 2724

2698 - 2658

2652 - 2623

2605 - 2605

2864 - 2806

2779 - 2770

2761 - 2717

2708 - 2618

2611 - 2595

2594 - 2582

Mortuary Temple of Shepseskaf at South Saqqara

Historical Range

C14 Mean age (weighted)

1 sigma

variance

probability

Calibrated Age BC
$2504-2500 \quad B C$ number used for mean: 7

$4146 \quad B P$

26

48 used for calibration

$3.5191 \%$
$17.21 \%$

ranges

one sigma $\quad 2865-2831$

$2821-2806$

2780 - 2770

$2761 \cdot 2717$

2710 - 2660

2650 - 2624

2878 - 2618

2611 - 2595

2594 - 2582 probability of range $\%$

19.0
8.5

5.5

25.0

93.6

3.9
2.5 
$5^{\text {th }}$ Dynasty (Old Kingdom)

South Pyramid Temple of Userkaf at Saqqara

Sample details: number dated: 6

$2498 \cdot 2491 \quad B C$

C14 Mean age (weighted)

1 sigma

Chi square

probability

Callbrated Age BC

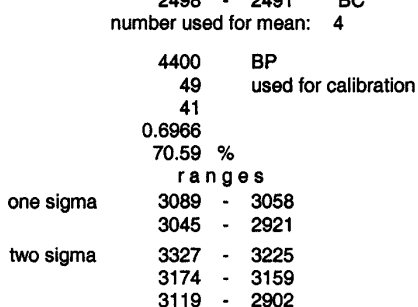

probability of range $\%$

19.9

80.1

13.8
1.8

$3119 \cdot 2902$

South Pyramid Temple of Userkaf at Saqqara

Sample details: two younger samples

C14 Mean age (weighted)

1 sigma

variance

Chi square

probability

Calibrated Age BC

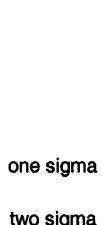

$3805 \quad B P$

70 used for calibration

50
0.4988

0.4988

$77.93 \%$

ranges

2398 - 2383
2346 - 2139

two sigma

$2461 \cdot 2115$

2099 - 2038

84.4

Pyramid of Userkaf at Saqqara

Historical Range

2498 - $2491 \quad B C$

Sample details: number dated: 7

C14 Mean age (weighted)

1 sigma

variance

Chi square

probability

Callbrated Age BC

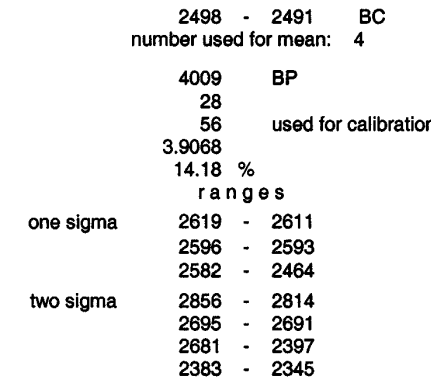

probability of range $\%$

5.5

94.5

91.0

probability of range $\%$

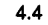

1.6

94.0

3.7

0.2

92.6
3.5

Pyramid of Userkaf at Saqqara

Sample details: three intrusive younger samples

C14 Mean age (weighted)

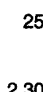

$2512 \quad$ BP

1 sigma

Chisquare

probability

Calibrated Age BC

$\begin{array}{crl} & 2512 \quad \text { BP } \\ & 30 \quad \text { used for calibration } \\ & 46 \quad \\ & 2.3001 \\ & 31.66 \% \\ \text { r a } \mathrm{ng} \text { e s } \\ \text { one sigma } \\ 787=757 \\ & 695=541 \\ \text { two sigma } & 796=502 \\ & 490=484 \\ & 464=449 \\ & 440=427 \\ & 423=413\end{array}$

probability of range \%
15.8
84.2
93.8
0.8
2.1
1.8
1.4


Queen's Pyramid of Userkaf at Saqqara Historical Range

C14 Mean age (weighted)

1 sigma

variance

Chi square

probability

Calibrated Age BC

qqara
one sigma
two sigma

probability of range $\%$

100.0

3.3

85.8

Mortuary Temple and Pyramid of Sahure at Abusir

Historical Range

Sample details: number dated: 14

C14 Mean age (weighted)

1 sigma

Chi square

probability

Calibrated Age BC

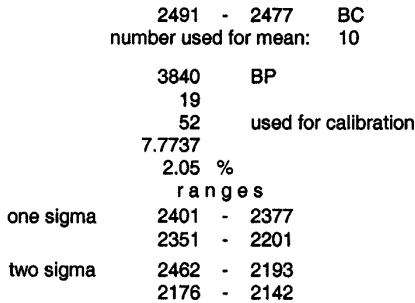

probability of range $\%$

12.9

87.1

93.9
6.1

Mortuary Temple of Unas at Saqqara

Historical Range

Sample details: number dated: 6

C14 Mean age (weighted)

1 sigma

variance

probability

Calibrated Age BC

one sigma

2375 - $2345 \quad B C$ number used for mean: 6

4009 BP

23 used for calibration

22

0.9699

$61.57 \%$

$\mathrm{rang}$ es
$2566-2546$

2566 - 2546
$2545-2520$

2498 - 2488

2479 - 2474

two sigma

2575 - 2509

$2504-2469$

probability of range $\%$

33.2

41.6

16.7

67.8
32.2

Pyramid of Unas at Saqqara Historical Range

Sample details: number dated: 5

C14 Mean age (weighted)

1 sigma

variance

Chi square

probability

Callbrated Age BC

$2375-2345 \quad B C$

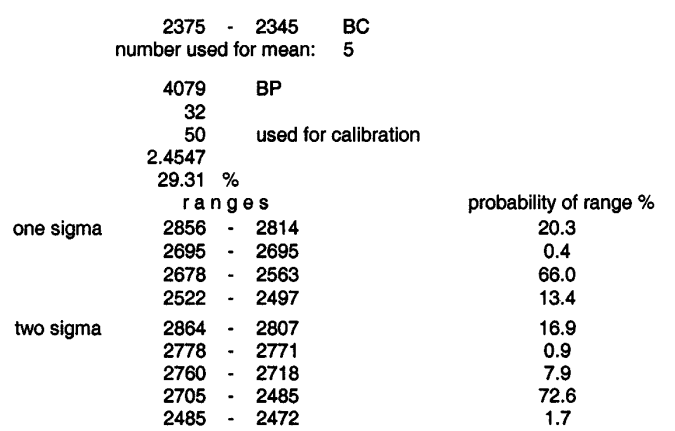


Tomb A, reign of Unas at Saqqara

Historical Age not established

Sample details: number dated: 2

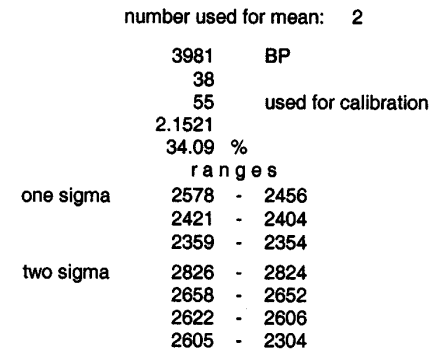

probability of range \%

Mean age (weighted)

1 sigma

Chi square

probability

Callbrated Age BC

$\begin{array}{ll}\text { one sigma } & 2578-2456 \\ & 2421=2404 \\ \text { two sigma } & 2359-2354 \\ & 2826-2824 \\ & 2658=2652 \\ & 2622=2606 \\ & 2605=2304\end{array}$

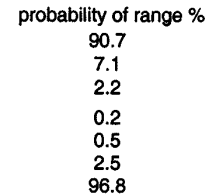

$6^{\text {th }}$ Dynasty (Old Kingdom)

Pyramid of Teti at Saqqara
Historical Range
Sample details: number dated: 15
C14 Mean age (weighted)
1 sigma
variance
Chi square
probability
Callbrated Age BC

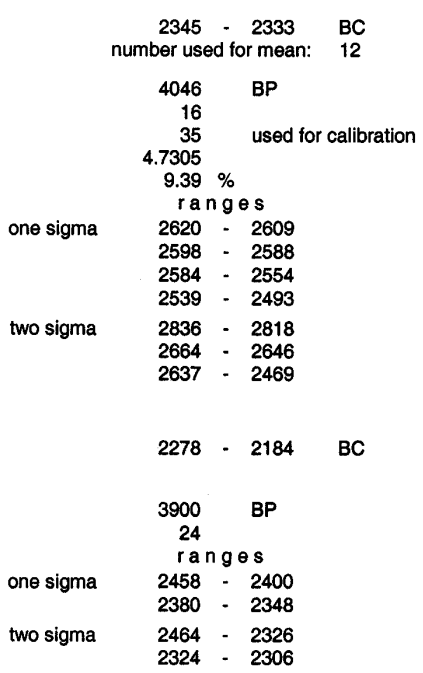

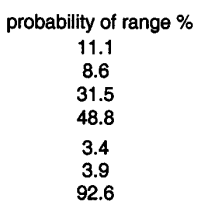

Pyramid of Pepi II at Saqqara Historical Range Sample details: number dated: 1

C14 Age of single sample date 1 sigma

Calibrated Age BC

$2324-2306$

probability of range \%
64.5
35.5
92.9
7.1

\section{$8^{\text {th }}$ Dynasty (First Intermediate Period)}

Pyramid of Qakare-lby at South Saqqara

Historical Range
Sample details: number dated: 1

$2181 \cdot 2161$

BC

C14 Age of single sample date

1 sigma

Callbrated Age BC

\begin{tabular}{|c|c|c|}
\hline & $\begin{array}{r}3872 \\
54 \\
\text { r a r }\end{array}$ & $\begin{array}{r}B P \\
g \in s\end{array}$ \\
\hline one sigma & $\begin{array}{l}2457 \\
2414\end{array}$ & $\begin{array}{l}2415 \\
-\quad 2289\end{array}$ \\
\hline $\mathrm{ma}$ & $\begin{array}{l}2471 \\
2161\end{array}$ & $\begin{array}{l}-\quad 2198 \\
-\quad 2149\end{array}$ \\
\hline
\end{tabular}

probability of range \%
23.9
76.1
98.8
1.2




\section{$12^{\text {th }}$ Dynasty (Middle Kingdom)}

\section{Pyramid of Senusret II at Illahu Historical Range Sample details: number dated: 9 \\ C14 Mean age (weighted) \\ 1 sigma \\ variance \\ Chi square \\ probability \\ Calibrated Age BC}

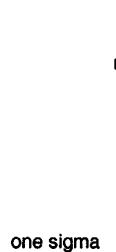

two sigma

Pyramid of Amenemhet III at Dashur Historical Range

Sample details: number dated: 2

C14 Age of single sample date

1 sigma

Calibrated Age BC
$1897-1878 \quad B C$ number used for mean: 8

3552 BP

20 used for calibration

0.17

0.6893
$70.85 \%$

ranges

$1935-1934$

1839 - 1829

$1785-1785$

$1949-1874$

$1843-1810$

$1800-1776$

$1842-1797 \quad B C$

number used for mean: 1

3442 BP

41

ranges

1864 - 1843

$1864=1843$

$1774-1688$

$1880-1837$

$1831 \div 1680$

$1670-1658$ probability of range $\%$

2.1
79.4

17.5

1.0
70.2

19.5

10.3

probability of range $\%$

16.9

4.7

76.0

2.4 